\title{
Providing a multi-objective scheduling tasks by Using PSO algorithm for cost management in cloud computing
}

\author{
Masrour Dowlatabadi, \\ Young Researchers and Elite \\ Club, Sabzevar \\ Branch, Islamic Azad University, \\ Sabzevar, Iran \\ Rasoul Habibzadeh Shojaee, \\ Department of Computer \\ Software, Central Islamshahr \\ Branch, Islamic Azad \\ University, Iran
}

Arman Kavosi Ghafi

Department of Computer

Software, Central Islamshahr

Branch, Islamic Azad

University, Iran

\author{
Abdolreza Andalib \\ Department of Computer \\ Software, Qeshm international \\ Branch, Islamic Azad \\ University, Qeshm, Iran
}

\begin{abstract}
:
This article is intended to use the multi-PSO algorithm for scheduling tasks for cost management in cloud computing. This means that any migration costs due to supply failure consider as a one objective and each task is a little particle and recognize by use of the appropriate fitness schedule function (how the particles arrangement) that cost at least amount of total expense. In addition to, the weight is granted to the each expenditure that reflects the importance of cost. The data which is used to simulate proposed method are series of academic and research data that are prepared from the Internet and MATLAB software is used for simulation. We simulate two issues, in the first issue, consider four task by four vehicles and divide tasks. In the second issue, make the issue more complicated and consider six tasks by four vehicles. We write PSO's output for each two issues of various iterations. Finally, the particles dispersion and as well as the output of the cost function were computed for each part.
\end{abstract}




\section{INTRODUCTION}

The basic concept of cloud computing dates back to 1995, When John McCarthy stated: Cloud computing may someday be organized as a public industry [1]. Cloud computing, briefly Consist of required computing, provide required fields and presenting computer services by a cloud of software, hardware and IT resources [2] [3]. Usually diagram of computer networks, the figure of the cloud is used as a summery way to hide the complex infrastructures that are inside the network. Cloud word in the cloud computing is extracted of these metaphor and is used to hide the Internet and infrastructures in the cloud computing [4]. By the cloud computing people use the PC to achieve a network of computers that are invisible status [5]. What makes this concept a scalable and efficient is a Service-Oriented Architecture (SOA) [6] [7]. Resources, are kept on servers providers instead of to be installed on a user's system.

By assume cloud computing, develop cloud concept, in addition to servers cover infrastructures of network. In mid-2008, Gartner found that there was an opportunity in cloud computing that was created for «Shaping the relationship between the consumers of IT services, between those who consume these services and those who sell these services ». That said, the cloud computing, will be the foundation in the IT industry for the next 20 years [8].Cloud computing is a new technology, which in recent decades by various companies, most notably Microsoft, Google and Amazon have been released to the world. Cloud computing technology is a great and safe storage space, which is always different with processing and computing power. In this regard, different companies, offered different services with various computing services [9]. These calculations are based on TCP / IP (internetbased) and include processors, massive memories, quick data transfer networks and architecture of reliable systems and without the governing standard network protocols, cannot be brought the entity to the technology [10]. Different types and levels of cloud services mean it is important that, we are working with which cloud computing systems [11]. A workflow, is an automation of processes to work on the documents, information, and tasks that passes from one process to another and usually is modeled in the form of a circular oriented graph (DOG) [12]. An example of this oriented graph can be seen in Figure 1. So that, each node shows a task and each edge shows dependencies between functional application tasks. Simply, the Process of mapping tasks in a workflow computing resources to perform tasks (To maintain dependencies between them) is defined workflows scheduling. The correct scheduling can have a great effect on system performance. The mapping of tasks to computing resources belonging to the class of things that are wellknown NP-Complete [12] [13]. Useful scheduling algorithm can meet user requirements and improve resource efficiency. The technology of cloud computing can be measured like telephone and electricity companies and sharing with customers [14]. Currently, Microsoft's cloud-based service share with customers as Microsoft Online service [15].Cloud computing models resources, similar to a public industry (electricity, telephone, etc.). As without the preparation and storage of expensive tanks, capacitors or transformers. Only when needed, we use electricity and water and only pay based on our consumption. In the case of software and other resources, we just open the software $(\mathrm{Eg}$, tap) and only pay the amount that we consumed. Without need to purchase and maintain expensive resources for their implementation [16]. The billing is based on the amount of minutes or hours that tenants spent on a cloud system, transferred data or filled storage space [17]. Sadri et al (1390) in his study that called "secure message transfer protocol in the cloud computing- based network". 
A brief description of a secure messaging system design under internal network according to the cloud computing model with utilizing Rayndl algorithms [18]. Ashoorion (1390) studied messages safety by computing in smart platforms.

He indicated that mobile agents architecture has overcome the many of limitations of architecture on client and server [19]. Delavar Ghorbannia and Arian (1390), provide a combined scheduling method based on genetic algorithm in cloud computing environment [20]. Matin Far (1391) were studied methods, challenges and tools to implement fault tolerance in cloud computing. In this research, the fault tolerant techniques in forms of cloud computing, fault tolerance policies, challenges and the tools used to implement fault tolerance in cloud computing were discussed[21]. Maleki et al (1391) in his study of the CloudSim's simulation model and it's evaluation, introduced CloudSim's simulation tools, that, enables modeling and simulation of cloud computing systems and environments to the preparation of applications [22]. Reza Zadeh (1391) in his study investigated "the tolerability of a bug in the cloud computing".

In these calculations some bug's tolerance models were presented. In these models redundancy has been used to improve the reliability [11]. Shams and Ghiyasi (1392), provided combined scheduling method based on genetic algorithm in cloud computing environment. In this study, they selected the distributed scheduling reliable algorithm in cloud computing (RSDC) due to its simplicity and has been investigated the reliability on the chosen algorithm [23]. Slvarany and Soodha (2010), offered a fee-based algorithms for efficient mapping of tasks to available resources in the cloud. The scheduling algorithm also measures the cost of using the resource and performance of analysis [24]. Raphael et al. (2011) is to minimize power consumption in data centers and improve their weight balance at the same time. In this regard, they stated a scheduling algorithm [25]. Moun and Tou (2011), provided an efficient method for scheduling virtual machine to virtual machine management. The proposed method has been called EVMS. Based on EVMS, scheduler system knows the current status of virtual machines in the cloud [26]. Huang et al. (2012) provided an efficient energy scheduling algorithm (ESS) to reduce energy consumption.

The results show that, ESS has ability to reduce energy consumption significantly by taking SLA's [27]. Huh et al. (2012), provided a task migration algorithm based virtual execution time that limited the maximum difference in virtual execution time between tasks. [28]. Peng et al. (2011), delivered an energy-efficient scheduling method based private cloud. In the paper presented three schedule criteria proposed and provided a combined of energy-efficient scheduling method [29]. The results show that, the efficiency of resources in the enhanced algorithm improved [30].

\section{Optimization Particles Algorithm}

The swarm particles optimization algorithm is one of the most important algorithms in the field of swarm intelligence. The algorithm introduced by Kennedy and Eberhart in 1995 and by inspiration of the social behavior of animals such as fish and birds live together in small and large groups, have been designed. Other names of this algorithm are the swarm particle algorithm and birds algorithm. PSO, has similarities with evolutionary computation techniques, like genetic algorithms (GA). However, unlike GA, PSO has not evolutionary functions, such as transit and crossover. For successful implementation of PSO, one of the key actions is, discover solution of the problem within the PSO 
particles. Correctly affects its performance and its possible implementation (Abraham et al., 2006). Kennedy and Eberhart (1995), demonstrated that PSO of each particle represents a possible answer that moves randomly in problem space. Change the location of each particle in the search space is influenced by its knowledge and neighbors, so the position of the swarm particle affects the way of particle search. As a result of modeling this behavior is the search process in which particles move to the appropriate areas. Particles are taught in groups, based on acquired knowledge, they go to the best of their neighbors. PSO based on the assumption that in every moment of every particle in the search space adjusts its location according to its best location, which has ever been and the best place in the neighboring. The overall design effective implement of PSO is difficult. Also, check out the dynamic environment for real-time applications such as fast convergence feature PSO to follow the target variable (minimum or maximum fitness function) are necessary.

\section{The proposed algorithm and simulation}

If you want to run your program in parallel and distributed in the cloud. We should know the properties of the environment. As we become more familiar with the characteristics of this environment, we will be able to use it better and more correct and avoid wasting resources. Parallelization of a program can be done at different levels. From the hardware to a single or multiple systems or cluster. Cloud computing architecture has a three-layer architecture which in the infrastructure layers, we are faced with resource management, the base layer is for development of applicable programs on the cloud resources and the software layer provided applications for final users. If we want to improve the management of resources, doing things and scheduling, we must go into the infrastructure layer. If we work on the development of a special issue generally, we should go into the substrate layer, and if we want to focus on how to provide final service for users, we have compiled software layer. Here we focused on the substrate layer, because we are going to develop a way to deal with cloud PSO algorithm. Our Intended use is in the field of scheduling tasks that can be as a final service is available to users and run and optimization is done in the substrate layer.

To be able to run the program in the field of PSO algorithm, it is necessary to provide the substrate layer for programs implement and development. The proposed method evaluation have been carried out by using two tests. we simulate PSO algorithm for the cloud network with 4 and 4 tools .Data by the network as follows:

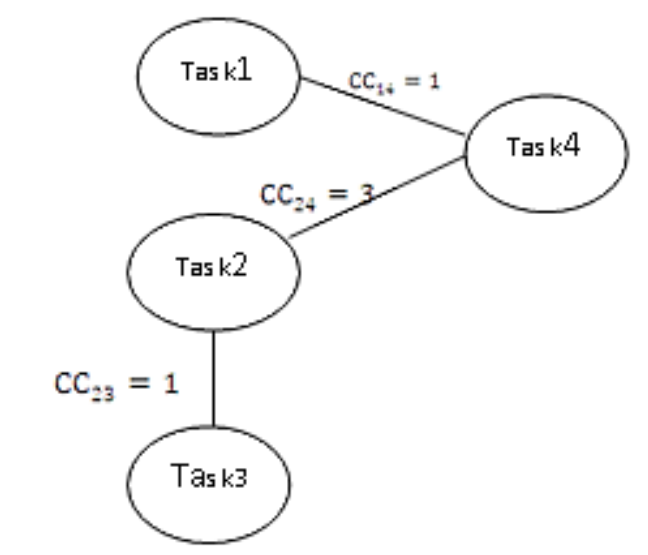

Figure 1. The task dependency graph

Duty as a TDG (task dependency graph) are shown in the following graph. The graph can be converted to the tasks matrix. 
Table 1. Tasks Matrix

\begin{tabular}{|l|l|l|l|l|}
\hline 4 & 3 & 2 & 1 & Task\# \\
\hline 1 & 0 & 0 & 0 & 1 \\
\hline 3 & 1 & 0 & 0 & 2 \\
\hline 0 & 0 & 1 & 0 & 3 \\
\hline 0 & 0 & 3 & 1 & 4 \\
\hline
\end{tabular}

Table 4.2 Price matrix task dependencies

\begin{tabular}{|l|l|l|l|l|}
\hline 4 & 3 & 2 & 1 & $\begin{array}{l}\text { Data } \\
\text { Center\# }\end{array}$ \\
\hline 4 & 2 & 1 & 0 & 1 \\
\hline 1 & 3 & 0 & 1 & 2 \\
\hline 2 & 0 & 3 & 2 & 3 \\
\hline 0 & 2 & 1 & 4 & 4 \\
\hline
\end{tabular}

The cost function is defined as follows:

Minimize Total costs $=\mathrm{f}(\mathrm{x})=\mathrm{TM}+\mathrm{TD}+\mathrm{TO}+\mathrm{TE}$ $+\mathrm{TC}$

$$
\mathrm{TC}=\sum \boldsymbol{C} \boldsymbol{j} \boldsymbol{i}+\boldsymbol{D} \boldsymbol{c k l}
$$

Where $\quad 1 \leq \mathrm{j} . \mathrm{i} \leq \mathrm{T}, \quad 1 \leq \mathrm{k}, \mathrm{l} \leq \mathrm{R}$

\begin{tabular}{|l|l|l|l|l|}
\hline & First & $\begin{array}{l}\text { Third } \\
\mathrm{e}\end{array}$ & $\begin{array}{l}\text { Focond } \\
\text { machin } \\
\mathrm{e}\end{array}$ & $\begin{array}{l}\text { machin } \\
\mathrm{e}\end{array}$ \\
$\begin{array}{l}\text { machin } \\
\mathrm{e}\end{array}$ \\
\hline $\begin{array}{l}\text { Wor } \\
\mathrm{k}\end{array}$ & 3 & 4 & 1 & 2 \\
\hline
\end{tabular}

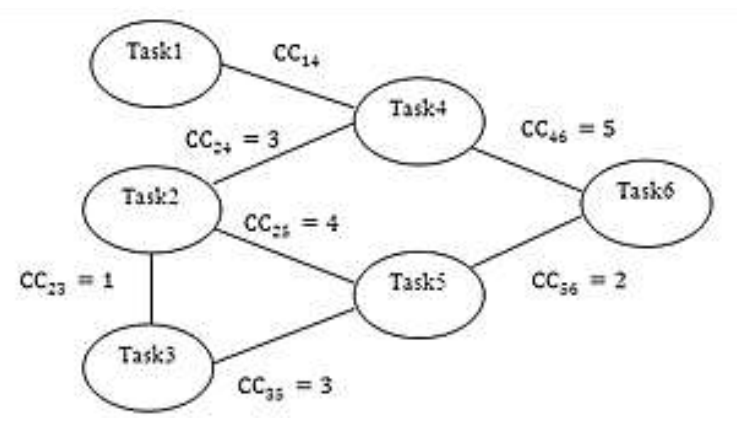

Cc, Dc are matrix elements tasks and dependent costs.

In this part, we optimize the cost function by using pso algorithm and the way of performance of each task for each car is determined to have the lowest cost. To convert this issue to a format which PSO could run it, we shoul define the limitation exactly to get correct results, So, in the next step, at least each device have a work, not in such way that many work are done by a device and another device does not have any work. For this part, we specify constraint for costs that is acceptable answer which every works will be divided equally between all equipment and correct answers should be also determined in the cost function.

The objective function described in this section. The calculation of the objective function is described below. Imagine we want to shift the task of machine number 2 to machine number 3 , rows and columns of matrix hould be moved in a way which Each task is assigned to the correct machine. In this particular case, row 2 to row 3 is replaced and column 2 and the third column are obtained at the end of a diagonal matrix in this case which confirms the correctness of operations.

After this step, we calculate the cost for each machine which is the product of two tasks and dependent costs matrixes. We have used PSO for aforementioned example, The results after 20 times as follows which final composition shows 
that the work assignement to the machine according to the cost function is as follows:

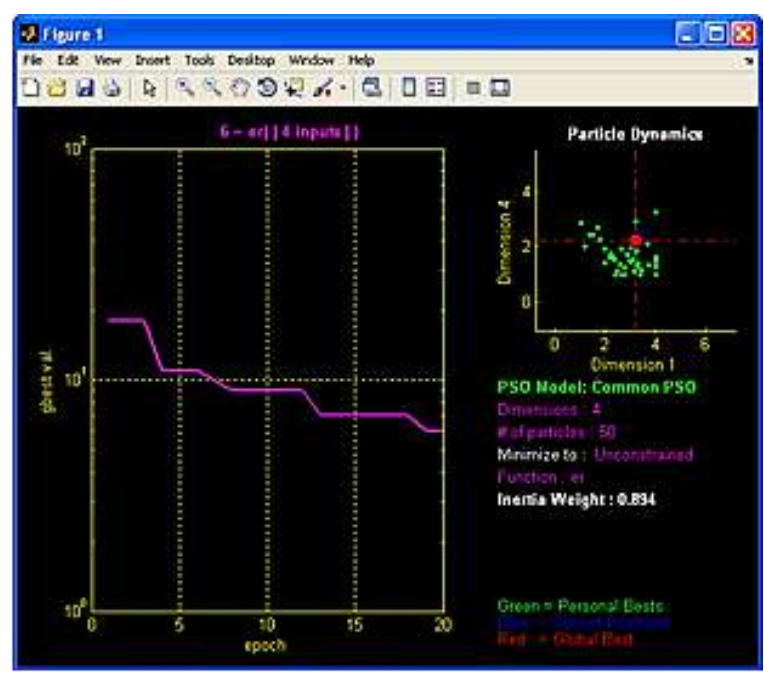

Figure 2 PSO output for the first case

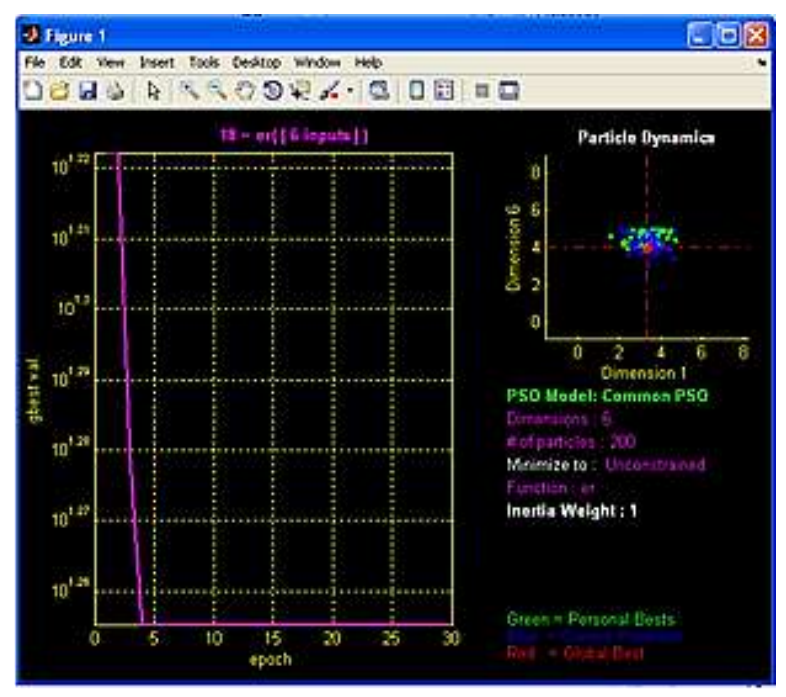

Figure 3 PSO output for the second case

\begin{tabular}{|l|l|l|l|l|l|l|}
\hline & First & $\begin{array}{l}\text { Thir } \\
\text { mac } \\
\text { hine }\end{array}$ & $\begin{array}{l}\text { Four } \\
\text { ond } \\
\text { mac } \\
\text { hine }\end{array}$ & $\begin{array}{l}\text { mac } \\
\text { mine } \\
\text { hift } \\
\text { mac } \\
\text { hine }\end{array}$ & $\begin{array}{l}\text { mac } \\
\text { hine }\end{array}$ & $\begin{array}{l}\text { h mac } \\
\text { hine }\end{array}$ \\
\hline $\begin{array}{l}\text { W } \\
\text { or } \\
\text { k }\end{array}$ & 3 & 4 & 1 & 2 & 2 & 4 \\
\hline
\end{tabular}

There is a condition in this case that must be met for the following:

Each machine can accept a maximum of two works

All machines must have partners to carry out the work.

The objective function can be expressed as before, except that dependent costs matrix is $4 \mathrm{x}$ 4 , When it would be expressed in terms of works, it needs to become a 6 x 6 matrix. For this conversion, It is necessary to repeat a column of the matrix twice and Or in other words, the rows and columns of the matrix which allocated to a machine that can do two things at the same time, is repeated twice and The general matrix is obtained. In this case, the simulation was conducted and the following results were obtained. Pso which have been used exerted initial population of 100 particles and we determined the number of repetitions on PSO, the amount of 30 was considered for repetitions.

\section{Conclusion}

According to the simulation results and adapting to the reality of tasks division can be concluded that PSO can properly diagnose the tasks division in cloud computing according to cost functions and graphs and for the division of tasks provides a good answer.

\section{Refrence}

[1] S.Pandey,L.Wu,S.M.Guru,andR.Buyya, A Particle Swarm Optimization-based Heuristic for Scheduling Workflow Applications in Cloud Computing Environments, peoc. On th IEEE International Conference on Advanced Information Networking and Applications, 2010. 
[2] Junjie Peng; Zhou Lei; Wu Zhang, An Energy-efficient Scheduling Approach Based on Private Clouds, Journal of Information \& Computational Science 8: 4 (2011).

[3] M. Damshenas, et al., "Forensics investigation challenges in cloud computing environments," in Cyber Security, Cyber Warfare and Digital Forensic (CyberSec), 2012 International Conference on, 2012, pp. 190-194.

[4] Y. Song, H. Wang, Y. Li, B. Feng, Y. Sun," Multi-Tiered On-Demand resource scheduling for VM-Based data center", in: Proceedings of the 9th IEEE/ACM International Symposium on Cluster Computing and the Grid , Shanghai, China,pp. 148-155 Object Management Group,2009

[5] M. Armbrust, A. Fox, R. Griffith, A.D. Joseph, R. Katz, A. Konwinski,"A view of cloud computing, Commun". ACM New York, USA,pp $50-58,2009$.

[6] G. Dhiman, K. Mihic, T. Rosing, "A system for online power prediction in virtualized environments using gaussian mixture models", in: Proceedings of the 47th ACM/IEEE Design Automation Conference,Anaheim, USA, pp. 807-812,2010.

[7] Ramgovind S, Eloff MM, Smith E, 2010, "The Management Of Security in Cloud Computing", IEEE.

[8] C. Clark, K. Fraser, S. Hand, J.G. Hansen, E. Jul, C. Limpach, I. Pratt and A. Warfield, "Live Migration of Virtual Machines", In Proc. of the second USENIX Symposium on Networked.

[9] David Burford, CFA, MBA, MCP Cloud Computing: A Brief Introduction February 20, 2010 .

[10] Gong, Ch., Liu, J., Zhang, Q., Chen, H., and Gong, Zh. 2010. The Characteristics of Cloud
Computing. 39th International Conference on Parallel Processing Workshops.

[11] Saynsky, Barry (1390), compete reference of cloud computing, Translated by Navid Farokhi, Computer Sciences Publication, First Version, Fall 1390

[12] T. Wei-Tek, et al., "Service-Oriented Cloud Computing Architecture," in Information Technology: New Generations (ITNG), 2010 Seventh International Conference on, 2010, pp. 684-689.

[13] Rajkumar Buyya, Chee Shin Yeo, Srikumar Venugopal,James Broberg, and Ivona Brandic."Cloud Computing and Emerging IT Platforms:Vision, Hype, and Reality for Delivering Computing as the 5 th Utility".Future Generation Computer Systems, , Amesterdam,The Netherlands,pp 599-616, June 2009.

[14] M. Armbrust, A. Fox, R. Griffith, A.D. Joseph, R. Katz, A. Konwinski,"A view of cloud computing, Commun". ACM New York, USA,pp 50-58,2009.

[15] Ghazizadeh, "Cloud Computing Benefits and Architecture in E-Learning," in Wireless, Mobile and Ubiquitous Technology in Education (WMUTE), 2012 IEEE Seventh International Conference on, 2012, pp. 199-201.

[16] Nurmi, D., Wolski, R., Grzegorczyk, C., Obertelli, G., Soman, S., Youseff, L., and Zagorodnov, D. 2008. Eucalyptus opensource cloudcomputing system. In CCA08: Cloud Computing and Its Applications.

[17] K. R. Joshi, et al., "Dependability in the cloud: Challenges and opportunities," in Dependable Systems \& Networks, 2009. DSN '09. IEEE/IFIP International Conference on, 2009, pp. 103-104. 
[18] Buyya, R., Yeo, C.S., Venugopal, S., Broberg, J., and Brandic, I., 2009. Cloud computing and emerging IT platforms: Vision, hype, and reality for delivering computing as the 5th utility. Future Generation Computer Systems, (2009), 25 (6), pp. 599-616.

[19]C. Clark, K. Fraser, S. Hand, J.G. Hansen, E. Jul, C. Limpach, I. Pratt and A. Warfield, "Live Migration of Virtual Machines", In Proc. of the second USENIX Symposium on Networked Systems Design and Implementation (NSDI), Boston, MA, USA, May 2005.

[20]Chunming Rong, Son T. Nguyen, Martin Gilje Jaatun, 2012, "Beyond Lighting: Asurvey on security challenges in cloud computing", Elsevier.

[21] D. Zissis and D. Lekkas, "Securing eGovernment and e-Voting with an open cloud computing architecture," Government Information Quarterly, vol. 28, pp. 239-251, 2011.

[22] David Burford, CFA, MBA, MCP Cloud Computing: A Brief Introduction February 20, 2010 .

[23] Amrhein, D., Forget Defining Cloud Computing.Available[Online]:http://ibm.ulitzer. com/node/10188.

[24] Ramgovind S, Eloff MM, Smith E, 2010, "The Management Of Security in Cloud Computing", IEEE.
[25] M. T. B. Aun ,et al., "Cloud enabled spam filtering services: Challenges and opportunities," in Sustainable Utilization and Development in Engineering and Technology (STUDENT), 2011 IEEE Conference on, 2011, pp. 63-68.

[26] Gong, Ch., Liu, J., Zhang, Q., Chen, H., and Gong, Zh. 2010. The Characteristics of Cloud Computing. 39th International Conference on Parallel Processing Workshops

[27] P. Mell and T. Grance. (2009, Oct.). The NIST definition of cloud com-puting, NIST special publication 800-145, National Institute of Standards and Technology. Available: http://www.csrc.nist.gov/publications/nistpubs/8 00-145/SP800-145.pdf.

[28] S. Chungman, et al., "Implementation of Cloud Computing Environment for Discrete Event System Simulation using Service Oriented Architecture," in Embedded and Ubiquitous Computing (EUC), 2010 IEEE/IFIP 8th International Conference on, 2010, pp. 359-362.

[29] Ivanka Menken , Gerard Blokdijk."Cloud Computing Virtualization Specialist Complete Certification Kit".2008.

[30] S. Marston, et al., "Cloud computing — The business perspective," Decision Support Systems, vol. 51, pp. 176-189, 2011 